\section{Estrutura e qualidade assistencial dos prestadores de serviços hospitalares à saúde suplementar no Brasil}

\author{
Healthcare structure and quality among \\ inpatient care providers for health \\ plans in Brazil
}

\author{
${ }^{1}$ Escola Nacional de Saúde \\ Pública Sergio Arouca, \\ Fundação Oswaldo Cruz, Rio \\ de Janeiro, Brasil. \\ 2 Escola Nacional de Ciências \\ Estatísticas, Rio de Janeiro, \\ Brasil. \\ Correspondência \\ M. C. Portela \\ Departamento de \\ Administração e \\ Planejamento em Saúde, \\ Escola Nacional de Saúde \\ Pública Sergio Arouca, \\ Fundação Oswaldo Cruz. \\ Rua Leopoldo Bulhões 1480, \\ Rio de Janeiro, $R J$ \\ 21041-210, Brasil. \\ mportela@ensp.fiocruz.br
}

Margareth Crisóstomo Portela 1

Sheyla Maria Lemos Lima 1

Maria Alícia Dominguez Ugá 1

Silvia Gerschman 1

Maurício Teixeira Leite de Vasconcellos 2

\section{Introdução}

This study aimed to characterize inpatient care providers for health plans in Brazil, considering that knowledge on healthcare providers is still incipient, particularly in relation to healthcare structure and quality. A national survey was performed, focusing on 3,817 hospitals. A stratified sample of 83 hospitals was selected, and data were collected from September to December 2006 using interviews with hospital administrators. Hospital care was characterized using variables related to installed capacity and services output, as well as practices and structures for healthcare quality assurance and control. The final sample consisted of 74 hospitals, representing an estimated universe of 3,799 hospitals. Inpatient care providers for health plans, mainly for the Unified National Health System (SUS), showed limited presence of structures and practices for improving management and healthcare quality.

Hospital Care; Quality of Health Care; Supplemental Health
A saúde suplementar no Brasil interage com um conjunto numericamente importante de hospitais privados que, simultaneamente, prestam serviços às operadoras de planos de saúde e ao Sistema Único de Saúde (SUS). Segundo o Cadastro Nacional de Estabelecimentos de Saúde (CNES; http://cnes.datasus.gov.br) do Ministério da Saúde, em julho de 2006, quando a pesquisa aqui apresentada iniciou, havia 6.343 hospitais no Brasil, dos quais $80,6 \%$ eram prestadores de serviços aos SUS, $60,5 \%$ prestavam serviços privados e 41,9\% atuavam nos setor público e privado. Todos esses hospitais contavam com aproximadamente 470 mil leitos, 39\% destes em hospitais estritamente prestadores de serviços ao SUS, $12 \%$ em hospitais estritamente prestadores de serviços privados e $49 \%$ em hospitais simultaneamente prestadores de serviços ao SUS e ao setor privado.

Nesse contexto, as operadoras de planos de saúde são responsáveis pela cobertura de cerca de $20 \%$ da população brasileira, incluindo 51,2 milhões de vínculos de beneficiários, estando aproximadamente $80 \%$ destes inscritos em planos de assistência médica 1. A partir de 1998 o Ministério da Saúde iniciou de forma mais incisiva a regulação dessas operadoras, constituindo, em 2000, com tal finalidade, a Agência Nacional de Saúde Suplementar (ANS), que vem empreendendo esforços para captar informações acerca 
dos usuários, da prestação da assistência e do funcionamento administrativo-financeiro do setor. Em 2004, instituiu o Programa de Qualificação de Saúde Suplementar, um sistema de avaliação das operadoras de planos de saúde, com o objetivo de induzir um processo contínuo de qualificação dos atores envolvidos (operadoras, prestadores, beneficiários e o próprio órgão regulador).

Uma das perspectivas presentes tem sido contribuir para a qualificação da gestão e da qualidade da atenção ofertada pelos prestadores de serviços assistenciais. No entanto, é ainda incipiente o conhecimento sobre tais prestadores de serviços de saúde, em particular sobre a sua estrutura e qualidade assistencial, bem como sobre os mecanismos e práticas relativos à qualificação da sua gestão. Dados assistenciais relativos ao setor de saúde suplementar são ainda escassos, centrando-se majoritariamente nas operadoras de planos de saúde, como unidades de observação.

Este trabalho objetivou caracterizar os prestadores de serviços hospitalares no setor de saúde suplementar, considerando a sua distribuição geográfica, porte, perfil e complexidade assistencial, relação com as operadoras de planos de saúde e o SUS, gestão e uso de práticas e instrumentos voltados para a melhoria contínua da qualidade assistencial.

\section{Métodos}

Esta pesquisa foi desenvolvida com base em um inquérito de abrangência nacional, caracterizando-se como um estudo transversal.

O universo do estudo, constituído por 3.817 hospitais prestadores de serviços às operadoras de planos de saúde, foi definido a partir do cruzamento do Cadastro de Prestadores de Serviços aos Planos de Saúde da ANS com o CNES em julho de 2006.

O cadastro foi estratificado por macrorregião e tipo de hospital (geral ou especializado), criando-se dez estratos naturais e alocando-se o tamanho da amostra de forma proporcional ao número de leitos. Em cada estrato natural, aplicou-se o algoritmo de Hedlin 2 para determinar o ponto de corte (número de leitos) entre um estrato certo (take-all) e um estrato amostrado (take-some); minimizou-se, dessa forma, a variância do número de leitos, dado um tamanho pré-fixado de amostra no estrato natural. Em alguns casos, no entanto, não foi necessário aplicar o algoritmo, por constarem menos de quatro hospitais no cadastro. O tamanho da amostra, inicialmente fixado em 75 hospitais, acabou sendo de 83 unidades por causa dos arredondamentos efetuados em cada estrato, sendo 28 certos e 55 amostrados.

Nos estratos amostrados, adotou-se um mecanismo de amostragem inversa 3,4 . Nesse método, as unidades da população são contatadas seqüencialmente a partir de uma ordem aleatória pré-definida, até que o número pré-fixado de entrevistas realizadas em cada estrato seja atingido. A definição da ordem de contato foi estabelecida pelo algoritmo de Hájek ${ }^{5}$. Os pesos amostrais foram calculados pelo inverso da probabilidade de inclusão de cada unidade na amostra em cada estrato, a fim de obter estimativas válidas para a população amostrada. Esta foi reduzida a 3.799 hospitais, pois 18 (7 certos e 11 em estratos amostrados) não pertenciam à população de pesquisa (só atendiam SUS) ou não puderam ser pesquisados (recusa em hospitais do estrato certo ou estratos onde todas as unidades foram contatadas sem que fosse atingido o tamanho de amostra pré-fixado).

A coleta de dados ocorreu entre setembro e dezembro de 2006, mediante entrevistas com os dirigentes das unidades hospitalares, realizadas por pesquisadores treinados. Em contato prévio à entrevista, já havia sido confirmado que o estabelecimento, de fato, prestava serviços hospitalares às operadoras de planos de saúde.

Para a caracterização assistencial dos hospitais, foram contempladas variáveis relativas não só à capacidade instalada e produção de serviços para planos de saúde e para o SUS, como também às práticas e estruturas de garantia e monitoramento da qualidade do cuidado. Algumas ferramentas de gestão da clínica tiveram destaque: diretrizes clínicas, entendidas como posicionamentos ou recomendações sistematicamente desenvolvidos para orientar médicos e pacientes acerca de cuidados de saúde apropriados, em circunstâncias clínicas específicas 6; gestão da patologia, que consiste no monitoramento do tipo de cuidado, resultados e custos relacionados a doenças selecionadas 7; gestão do caso, que envolve o monitoramento de resultados e custos relativos a clientes selecionados 7 .

As análises realizadas foram descritivas, tendo sido processadas por meio do programa estatístico SAS (SAS Inst., Cary, Estados Unidos), não havendo necessidade de tratamento de dados de amostras complexas para os resultados apresentados, que requereram, apenas, o uso dos pesos amostrais.

Para classificar os hospitais por porte e complexidade, foi utilizado um fluxo lógico, considerando-se as categorias existentes no CNES, os serviços médicos, equipamentos biomédicos e procedimentos hospitalares de alta complexidade reportados 8 . Foram construídas cinco classes 
de hospitais: classe Geral II, com hospitais gerais que obrigatoriamente registraram a presença de UTI e a realização de procedimentos de alta complexidade na internação, com equipamentos em uso compatíveis, nas áreas cardiovascular, cirurgia oncológica, neurocirurgia, ortopedia, transplantes ou neonatologia; classes Geral I com UTI e Geral I sem UTI, constituídas por hospitais gerais de menor complexidade e hospitais de clínicas básicas; classes Especializado com UTI e Especializado sem UTI - as categorias com UTI requereram o registro de UTI, bem como de leitos de cuidados intensivos e de equipamentos em uso compatíveis com este tipo de cuidado.

\section{Resultados}

Considerando as exclusões decorrentes de erros no cadastro (hospitais que não prestavam serviços no campo da saúde suplementar ou estabelecimentos não hospitalares) e as recusas no estrato certo, sem possibilidade de substituição e, em dois estratos amostrados, com listas-reserva esgotadas, a amostra final pesquisada resultou em 74 hospitais - 21 no estrato certo e 53 no estrato amostrado. O resultado final se constitui em um universo estimado de 3.799 hospitais, em contraponto ao universo programado de 3.817 hospitais.

\section{Localização geográfica, porte e} complexidade dos hospitais e relação com as operadoras de planos de saúde

Os prestadores de serviços hospitalares na saúde suplementar concentram-se predominantemente na Região Sudeste (41,5\%), seguida pelas regiões Sul (21,6\%), Nordeste (19\%), Centro-Oeste $(12,9 \%)$ e Norte $(5 \%)$. Como seria esperado, acompanham a distribuição das operadoras de planos de saúde no país: Região Sudeste $(59,5 \%)$, Sul (17\%), Nordeste (13\%), Centro-Oeste $(7,2 \%)$ e Norte $(3,3 \%) 9$.

Analisando-se a complexidade assistencial dos hospitais, observa-se que $56,1 \%$ caracterizam-se como de menor complexidade, segundo a classificação aqui utilizada, sendo $42 \%$ do tipo Geral I sem UTI e 14,1\% Especializado sem UTI. A outra parte $(43,9 \%)$ é constituída por hospitais de maior complexidade: Geral II, 22,8\%; Geral I com UTI, 17,9\%; Especializado com UTI, 3,2\%. Os hospitais especializados identificados foram pediátricos, de traumato-ortopedia, psiquiátricos e maternidades. Entretanto, tendo em vista o tamanho da amostra, não se pode afirmar que não existiam hospitais de outras especialidades no universo de prestadores hospitalares da saúde suplementar.

Na Tabela 1, observa-se a distribuição dos hospitais segundo sua complexidade nas regiões do país. Destaca-se que quase a totalidade dos hospitais de maior complexidade (Geral II) está localizada nas regiões Sul e Sudeste, onde também estão concentrados $76,5 \%$ das operadoras de planos de saúde.

Considerando-se o número de leitos clínicocirúrgicos ativos, 70,9\% dos hospitais apresentaram menos de cem leitos: 0-10 (5,7\%); 11-50 (37\%); 51-100 (28,2\%). Os demais se distribuíram da seguinte forma: 101-150 leitos (15,7\%); 151350 (13,1\%); > 350 (0,3\%).

Os hospitais de menor complexidade - Geral I sem UTI - corresponderam majoritariamente àqueles de menor porte de leitos, enquanto os de maior complexidade - Geral II - predominaram entre os de maior porte (Tabela 2).

A grande maioria dos hospitais (72\%) referiu prestar serviços ao SUS. Entre esses hospitais, o percentual médio de atendimento ao SUS foi de

Tabela 1

Distribuição dos hospitais prestadores de serviços na Saúde Suplementar, segundo complexidade e região do país. Brasil, 2006

\begin{tabular}{|c|c|c|c|c|c|c|c|c|c|c|c|c|}
\hline \multirow[t]{2}{*}{ Complexidade } & \multicolumn{2}{|c|}{ Norte } & \multicolumn{2}{|c|}{ Nordeste } & \multicolumn{2}{|c|}{ Sudeste } & \multicolumn{2}{|c|}{ Sul } & \multicolumn{2}{|c|}{ Centro-Oeste } & \multicolumn{2}{|c|}{ Total } \\
\hline & $\mathbf{n}$ & $\%$ & $\mathbf{n}$ & $\%$ & $\mathrm{n}$ & $\%$ & $\mathbf{n}$ & $\%$ & n & $\%$ & $\mathrm{n}$ & $\%$ \\
\hline Especializado com UTI & 10 & 5,6 & 1 & 0,2 & 110 & 7,0 & 0 & 0,0 & 0 & 0,0 & 122 & 3,2 \\
\hline Especializado sem UTI & 0 & 0,0 & 226 & 31,4 & 166 & 10,5 & 37 & 4,5 & 106 & 21,6 & 535 & 14,1 \\
\hline Geral I com UTI & 168 & 88,7 & 211 & 29,3 & 229 & 14,5 & 74 & 9,0 & 0 & 0,0 & 681 & 17,9 \\
\hline Geral I sem UTI & 11 & 5,7 & 140 & 19,4 & 837 & 53,0 & 223 & 27,2 & 384 & 78,2 & 1.595 & 42,0 \\
\hline Geral II & 0 & 0,0 & 142 & 19,7 & 237 & 15,0 & 486 & 59,3 & 1 & 0,2 & 866 & 22,8 \\
\hline Total & 189 & 100,0 & 720 & 100,0 & 1.579 & 100,0 & 820 & 100,0 & 491 & 100,0 & 3.799 * & 100,0 \\
\hline
\end{tabular}

* Refere-se ao total de unidades no cadastro de seleção após eliminação das unidades que não pertenciam à população de pesquisa. 
Tabela 2

Distribuição dos hospitais prestadores de serviços na saúde suplementar, segundo complexidade assistencial e porte de leitos. Brasil, 2006.

\begin{tabular}{|c|c|c|c|c|c|c|c|c|c|c|c|c|c|c|}
\hline \multirow[t]{3}{*}{ Complexidade } & \multicolumn{14}{|c|}{ Porte de leitos } \\
\hline & \multicolumn{2}{|c|}{$11-50$} & \multicolumn{2}{|c|}{$51-100$} & \multicolumn{2}{|c|}{$101-150$} & \multicolumn{2}{|c|}{$151-350$} & \multicolumn{2}{|c|}{$351-500$} & \multicolumn{2}{|c|}{$>500$} & \multicolumn{2}{|c|}{ Total } \\
\hline & $\mathbf{n}$ & $\%$ & $\mathbf{n}$ & $\%$ & $\mathbf{n}$ & $\%$ & $\mathbf{n}$ & $\%$ & $\mathbf{n}$ & $\%$ & $\mathrm{n}$ & $\%$ & $\mathbf{n}$ & $\%$ \\
\hline Especializado com UTI & 0 & 0,0 & 122 & 10,6 & 0 & 0,0 & 0 & 0,0 & 0 & 0,0 & 0 & 0,0 & 122 & 3,2 \\
\hline Especializado sem UTI & 205 & 16,8 & 0 & 0,0 & 110 & 14,7 & 219 & 36,3 & 0 & 0,0 & 1 & 9,1 & 535 & 14,1 \\
\hline Geral I com UTI & 148 & 12,2 & 226 & 19,8 & 294 & 39,5 & 12 & 2,1 & 0 & 0,0 & 0 & 0,0 & 681 & 17,9 \\
\hline Geral I sem UTI & 794 & 65,2 & 572 & 50,0 & 229 & 30,6 & 0 & 0,0 & 0 & 0,0 & 0 & 0,0 & 1.595 & 42,0 \\
\hline Geral II & 70 & 5,8 & 225 & 19,6 & 113 & 15,2 & 372 & 61,6 & 77 & 100,0 & 10 & 90,9 & 866 & 22,8 \\
\hline Total & 1.217 & 100,0 & 1.145 & 100,0 & 746 & 100,0 & 603 & 100,0 & 77 & 100,0 & 11 & 100,0 & 3.799 * & 100,0 \\
\hline
\end{tabular}

* Refere-se ao total de unidades no cadastro de seleção após eliminação das unidades que não pertenciam à população de pesquisa.

$74,5 \%$, com mediana de $80 \%$; o percentual médio de atendimento a operadoras foi de $19,7 \%$, com mediana de $16 \%$; por fim, o percentual médio de atendimento a particulares foi de $5,8 \%$, com mediana de $5 \%$.

Considerando o vínculo com as operadoras, $97,1 \%$ dos hospitais foram declarados credenciados e apenas $7 \%$, pertencentes a uma operadora, sublinhando-se que as duas alternativas não são excludentes. Em relação ao número de operadoras para as quais os hospitais prestam serviços, observou-se a seguinte distribuição: 1 operadora (6\%); $2-5$ operadoras $(25,5 \%) ; 6-10$ operadoras (18,8\%); $11-20$ operadoras (22\%); $21-50$ operadoras $(22,7 \%)$; > 50 operadoras $(4 \%)$; sem informação (1\%).

Para a maior parte dos hospitais $(79,1 \%)$, a UNIMED foi citada como uma das cinco principais operadoras em volume de faturamento. Outras operadoras referidas por número significativo de hospitais foram: CASSI (40,1\%), GEAP (27,2\%), Bradesco (19,5\%), Sul América (12,4\%), Correios (11,2\%), Cabesp (11\%), Polícia Militar (9\%) e IPERGS $(8,9 \%)$. Para $81,1 \%$ dos hospitais, as principais operadoras em volume de faturamento coincidiram com as principais em volume de internação. No que concerne ao percentual médio de participação das três primeiras operadoras em volume de faturamento, registrou-se: primeira operadora $52,3 \%( \pm 181,9 \%)$; segunda operadora $17 \%( \pm 81,6 \%) \%$; terceira operadora $7,9 \%( \pm 39,8 \%)$.

A Tabela 3 mapeia a existência e credenciamento de serviços por operadoras de planos de saúde nos hospitais estudados, sugerindo que serviços disponíveis podem, com alguma freqüência, ser ofertados somente para o SUS.

Dos hospitais que declararam pertencer a alguma rede de prestadores de operadoras $(46,5 \%)$,
$74,1 \%$ referiram possuir algum serviço de referência: urgência/emergência, 29,6\%; atenção à gestação e ao parto, 27,7\%; atenção cardiovascular, 23,7\%; atenção de alta complexidade, 23,5\%; cuidado intensivo, 23,1\%; atenção em oncologia, $15,3 \%$.

\section{Qualificação da gestão e monitoramento da qualidade}

A maior parte dos hospitais informou não ter as estruturas e práticas de qualificação da gestão consideradas e utilizadas em outro estudo para classificar o desenvolvimento gerencial de hospitais filantrópicos no Brasil 9. Como estruturas qualificadoras, consideraram-se a existência de serviços de epidemiologia, de qualidade e de ouvidoria, o hospital estar acreditado ou em processo, dentre outras. Como práticas, o funcionamento 24 horas do serviço de patologia clínica e de imagem, a dispensação de medicamento por dose individualizada e ou unitária, a existência de prontuário único e a capacitação de profissionais da assistência e da limpeza.

De forma diferenciada, o funcionamento ininterrupto do laboratório de patologia clínica e do serviço de imagem, condições estruturais de garantia da presteza e da qualidade da atenção, especialmente em hospitais com serviços de urgência/emergência, foi registrado em respectivamente $73,4 \%$ e $72,8 \%$ dos hospitais (Tabela 4). Merece ainda destaque a dispensação de medicamentos na modalidade de dose individualizada, em 51,5\% dos hospitais, considerada boa prática de assistência farmacêutica. Para as demais condições investigadas, menos da metade dos hospitais acusou sua existência.

Observa-se, ainda, que o fato de os hospitais prestarem serviços ao SUS não tem uma relação 
Distribuição de hospitais prestadores de serviços na saúde suplementar, segundo serviços existentes e credenciados por, pelo menos, uma de suas principais operadoras. Brasil, 2006.

\begin{tabular}{|c|c|c|c|c|}
\hline \multirow[t]{2}{*}{ Serviço } & \multicolumn{2}{|c|}{ Existente } & \multicolumn{2}{|c|}{ Credenciado } \\
\hline & n & $\%$ & $\mathbf{n}$ & $\%$ \\
\hline Internação clínica médica & 3.520 & 92,7 & 2.951 & 77,7 \\
\hline Internação cirurgia geral & 3.624 & 85,9 & 2.731 & 71,9 \\
\hline Internação obstetrícia & 2.887 & 76,0 & 2.354 & 62,0 \\
\hline Internação ginecologia & 3.164 & 83,3 & 2.561 & 67,4 \\
\hline Internação pediatria & 2.966 & 78,1 & 2.395 & 63,1 \\
\hline Internação traumato-ortopedia & 2.496 & 65,7 & 1.945 & 51,2 \\
\hline Centro cirúrgico & 3.458 & 91,0 & 2.817 & 74,2 \\
\hline Centro obstétrico & 2.736 & 72,0 & 2.204 & 58,0 \\
\hline Ambulatório & 3.080 & 81,1 & 2.552 & 67,2 \\
\hline UTI adulto & 1.610 & 42,4 & 1.292 & 34,0 \\
\hline UTI pediátrica & 649 & 17,1 & 573 & 15,1 \\
\hline UTI neonatal & 703 & 18,5 & 515 & 13,6 \\
\hline Unidade coronariana & 473 & 12,5 & 299 & 7,9 \\
\hline Unidade de queimados & 154 & 4,1 & 77 & 2,0 \\
\hline Unidade intermediária & 1.098 & 28,9 & 736 & 19,4 \\
\hline Emergência & 3.284 & 86,4 & 2.715 & 71,5 \\
\hline Hospital dia & 1.069 & 28,1 & 657 & 17,3 \\
\hline Atendimento domiciliar/Home care & 260 & 6,8 & 153 & 4,0 \\
\hline Laboratório de patologia/Análises clínicas & 3.509 & 92,4 & 2.926 & 77,0 \\
\hline Laboratório de anatomia patológica & 2.628 & 69,2 & 2.159 & 56,8 \\
\hline Raio-X & 3.278 & 86,3 & 2.752 & 72,4 \\
\hline Ultra-sonografia & 2.826 & 74,4 & 2.490 & 65,5 \\
\hline Tomografia & 1.288 & 33,9 & 1.199 & 31,6 \\
\hline Ressonância & 393 & 10,4 & 381 & 10,0 \\
\hline Hemodinâmica & 572 & 15,1 & 451 & 11,9 \\
\hline Angiografia & 646 & 17,0 & 487 & 12,8 \\
\hline Hemodiálise & 898 & 23,6 & 808 & 21,3 \\
\hline Medicina nuclear & 272 & 7,2 & 272 & 7,2 \\
\hline Hemoterapia & 1.589 & 41,8 & 1.172 & 30,9 \\
\hline Radioterapia & 23 & 0,6 & 11 & 0,3 \\
\hline Quimioterapia & 660 & 17,4 & 497 & 13,1 \\
\hline Psiquiatria & 218 & 5,7 & 218 & 5,7 \\
\hline
\end{tabular}

positiva com a presença de estruturas e práticas de qualificação da gestão. Ao contrário, dentre aqueles que não prestam serviços ao SUS, uma maior porcentagem conta com tais estruturas e práticas.

A maioria dos hospitais registrou a presença de comissão de controle de infecção hospitalar $(74,8 \%)$, comissão de revisão de prontuários $(61,9 \%)$ e comissão de ética médica $(60,3 \%)$, bem como a realização de pesquisa de satisfação do usuário $(75,4 \%)$ (Tabela 5). O maior problema evidenciado pelos resultados observados, entre- tanto, diz respeito à irregularidade de atuação dessas comissões/atividades.

No que se refere à presença de sistema de informação, condição estrutural sem a qual não é possível proceder à avaliação e ao monitoramento da qualidade assistencial, observa-se que $94,2 \%$ dos hospitais referiram possuir cadastro de pacientes, e $77,2 \%$, sistema de informação sobre a produção assistencial. Poucos hospitais registraram ter prontuário eletrônico em alguns serviços $(3,2 \%)$ e em todos os serviços $(3,7 \%)$. Adicionados ao fato de somente $36,1 \%$ dos hos- 
Distribuição dos hospitais prestadores de serviços na saúde suplementar, segundo presença de estruturas e práticas de qualificação da gestão ( $\mathrm{N}=3.799$ *). Brasil, 2006.

\begin{tabular}{|c|c|c|c|c|c|c|c|}
\hline \multirow[t]{3}{*}{ Estruturas e práticas de qualificação da gestão } & \multicolumn{2}{|c|}{ Total } & \multicolumn{4}{|c|}{ SUS } & \multirow{3}{*}{$\begin{array}{c}\text { Valor } \\
\text { de } p \text { ** }\end{array}$} \\
\hline & & & \multicolumn{2}{|c|}{ Sim } & \multicolumn{2}{|c|}{ Não } & \\
\hline & $\mathrm{n}$ & $\%$ & $\mathrm{n}$ & $\%$ & $\mathrm{n}$ & $\%$ & \\
\hline Serviço/Assessoria/Núcleo de epidemiologia & 928 & 24,4 & 591 & 21,6 & 338 & 31,7 & 0,5481 \\
\hline Serviço/Assessoria/Núcleo de qualidade & 1.484 & 39,1 & 566 & 20,7 & 918 & 86,1 & 0,0007 \\
\hline Ouvidoria & 1.420 & 37,4 & 698 & 49,2 & 722 & 67,7 & 0,0106 \\
\hline Acreditação & & & & & & & 0,4384 \\
\hline Em processo & 252 & 7,0 & 139 & 5,4 & 113 & 10,6 & \\
\hline Concluída & 224 & 6,1 & 80 & 3,1 & 144 & 13,5 & \\
\hline Com algum serviço certificado pelo ISO & 293 & 7,7 & 219 & 8,0 & 74 & 67,0 & 0,6197 \\
\hline Prêmio de qualidade & 1.601 & 42,2 & 1.068 & 39,1 & 534 & 50,1 & 0,4999 \\
\hline Membro de associação da área hospitalar & 3.612 & 95,1 & 2.546 & 93,2 & 1.066 & 100,0 & 0,1333 \\
\hline Funcionamento da patologia clínica 24 horas/7 dias por semana & 2.787 & 73,4 & 1.959 & 71,7 & 828 & 77,7 & 0,0501 \\
\hline Funcionamento da imagem 24 horas/7 dias por semana & 2.766 & 72,8 & 1.881 & 68,8 & 886 & 83,1 & 0,0246 \\
\hline Dispensação de medicamento (dose unitária/paciente) & 1.051 & 27,6 & 602 & 22,0 & 448 & 42,0 & 0,1629 \\
\hline Dispensação de medicamento (dose individual/paciente-dia) & 1.957 & 51,5 & 1.465 & 53,6 & 493 & 46,2 & 0,6069 \\
\hline Dispensação de medicamento (dose coletiva) & 617 & 16,2 & 562 & 20,6 & 55 & 5,2 & 0,1171 \\
\hline Organização de prontuários & & & & & & & 0,4182 \\
\hline Único para pacientes externos e internos & 1.371 & 36,1 & 905 & 33,1 & 466 & 43,7 & \\
\hline Separados para pacientes externos e internos & 1.522 & 40,1 & 1.144 & 41,9 & 378 & 35,5 & \\
\hline Capacitação de profissionais da assistência & 1.255 & 33,0 & 601 & 22,0 & 655 & 61,4 & 0,0422 \\
\hline Capacitação de profissionais da limpeza & 1.741 & 45,8 & 1.097 & 40,1 & 645 & 60,5 & 0,2331 \\
\hline
\end{tabular}

* Refere-se ao total de unidades no cadastro de seleção após eliminação das unidades que não pertenciam à população de pesquisa.

** Teste de $\chi^{2}$.

pitais terem referido possuir prontuário único para seus pacientes, esses dados sugerem baixa coordenação das atividades realizadas pelos diferentes profissionais sobre o paciente no decorrer do tempo, dificultando o alcance de uma atenção mais integral e de qualidade.

Em relação aos sistemas de registro da produção assistencial, observa-se que a grande parte dos hospitais utiliza indicadores tradicionais, mas que pouco expressam a qualidade assistencial. Os indicadores referentes à taxa de ocupação de leitos e tempo médio de internação foram registrados, respectivamente, por $84,4 \%$ e $86,4 \%$ dos hospitais. As taxas de infecção hospitalar e de mortalidade geral, que são indicadores mais próximos da qualidade assistencial, foram citadas, respectivamente, por $80,5 \%$ e $70,7 \%$ dos hospitais. Considerando-se, entretanto, alguns indicadores mais sofisticados, os percentuais diminuem. A taxa de suspensão de cirurgia foi citada por apenas $29,1 \%$ dos hospitais. Levandose em conta que $85,9 \%$ e $91 \%$ dos hospitais referiram ter, respectivamente, os serviços de inter- nação em clínica cirúrgica e o centro cirúrgico, poder-se-ia esperar uma taxa mais elevada de controle de suspensão de cirurgia. Além disso, somente $25,4 \%$ dos hospitais controlaram a taxa de mortalidade por algumas doenças específicas. O controle da reinternação pelo mesmo motivo foi acusado por somente $27,2 \%$ dos hospitais, e o controle de eventos adversos, por apenas $27,2 \%$ dos hospitais.

A inscrição dos laboratórios de patologia/ análise clínica dos hospitais a algum programa interlaboratorial pode ser considerada como um indicador da qualidade desses serviços. Observase, no entanto, que poucos hospitais acusaram estar associados a algum programa. Somente $38,4 \%$ dos hospitais referiram que seus laboratórios integravam programas conhecidos. Era esperado encontrar um percentual maior de associação, tendo em vista que $92,4 \%$ dos hospitais estudados referiram possuir o serviço de laboratório de patologia/análise clínica. 
Presença de estruturas e práticas de garantia e monitoramento da qualidade assistencial nos prestadores de serviços na saúde suplementar $(\mathrm{N}=3.799$ *). Brasil, 2006.

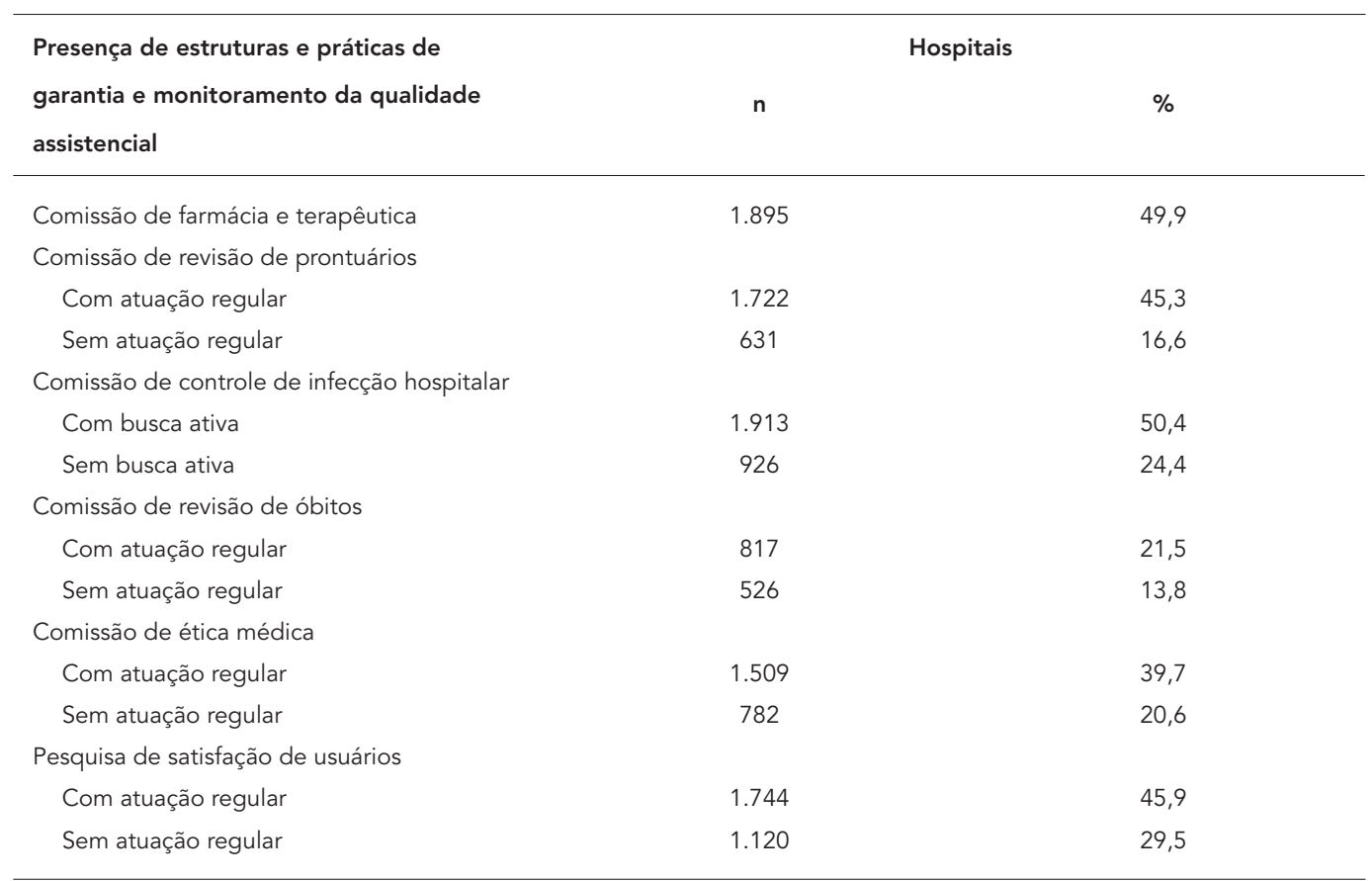

* Refere-se ao total de unidades no cadastro de seleção após eliminação das unidades que não pertenciam à população de pesquisa.

\section{Ferramentas e práticas da gestão da clínica}

Pouco mais da metade dos hospitais referiu utilizar segunda opinião para procedimentos específicos $(52,2 \%)$ e diretrizes clínicas $(51,6 \%)$; $30,5 \%$, gestão do caso; $24,2 \%$, gestão de patologias; $22,2 \%$, estudos estatísticos sobre variações da prática médica.

Verificou-se uma associação estatisticamente significante $(p<0,0001)$ entre o uso de diretrizes clínicas e complexidade hospitalar: especializado com UTI (100\%), Geral II (82,6\%), Geral I com UTI (78,6\%), Geral I sem UTI (29,9\%) e Especializado sem UTI (20,8\%).

Considerando as patologias/condições que são o alvo das diretrizes clínicas nos hospitais em que estas são adotadas, a mais freqüente foi infecção hospitalar (66,2\%), seguida de hipertensão arterial $(62,4 \%)$, assistência ao parto $(54,9 \%)$, diabetes $(54,4 \%)$, assistência ao recém-nato de alto risco $(52,4 \%)$ e infarto agudo do miocárdio $(52,1 \%)$.

No que tange às formas de divulgação das diretrizes clínicas entre seus profissionais, os hospitais que declararam utilizá-las reportaram as seguintes estratégias: seminários e palestras
(57,3\%), livretos (48,8\%), fluxogramas $(30,4 \%)$ e outras (45,1\%). A divulgação das diretrizes para pacientes foi ainda mais baixa, estando ausente em $66,7 \%$ dos hospitais pertinentes.

Na maior parte $(80,9 \%)$ dos $51,6 \%$ de hospitais com diretrizes clínicas, houve registro de alguma avaliação a respeito da adesão dos profissionais, realizada, de forma não excludente, por meio do acompanhamento de pedidos de exames, medicamentos ou procedimentos especiais $(52,1 \%)$ e da avaliação de prontuários (50,3\%).

As doenças ou condições mais focalizadas pela gestão de patologias nos hospitais que declararam realizá-la foram: atenção pré-natal e/ ou ao parto $(60,1 \%)$, infecção hospitalar $(53,2 \%)$ e hipertensão arterial (32\%). Chama atenção a baixa freqüência da gestão do diabetes (20\%), asma $(12 \%)$, câncer de mama e de próstata $(9,5 \%)$, infarto agudo do miocárdio (8,5\%), AIDS (8,3\%) e câncer de colo de útero (1,6\%).

Dos hospitais que referiram fazer gestão de patologias, 66,8\% declararam divulgar aos pacientes as intervenções nelas preconizadas. Contudo, apenas em 23,1\% desses hospitais houve o envolvimento da maioria dos pacientes 
na tomada de decisão sobre condutas clínicocirúrgicas. Em 24,2\% dessas unidades, somente a minoria dos pacientes foi envolvida, enquanto em $30,8 \%$ dos casos nunca ocorreu o envolvimento dos pacientes nessas condutas.

Os agravos e condições mais focalizados na gestão do caso foram o diabetes em estado avançado $(55,6 \%)$ e os idosos com múltiplas doenças $(55,5 \%)$.

Os critérios mais freqüentes para a seleção dos casos a serem geridos foram recorrência de internações $(66,5 \%)$, gravidade clínica, múltiplas doenças ou baixa adesão ao tratamento $(47,1 \%)$ e complicação clínica (46,3\%). Diferentemente do esperado, o alto custo foi um critério mencionado por apenas $21,2 \%$ dos hospitais.

Entre os hospitais que referiram envolver seus pacientes na tomada de decisão concernente à gestão de casos, somente $30,9 \%$ declararam sempre fazê-lo; $17,2 \%$ declararam fazê-lo na maioria das vezes e $22,9 \%$ declararam nunca fazê-lo. Quanto ao profissional que atua predominantemente como gestor do caso, o médico foi o mais freqüentemente $(64,2 \%)$ referido, seguido do enfermeiro, mencionado em $29,2 \%$ dos casos.

No que concerne aos critérios utilizados para a realização de estudos estatísticos sobre a variação da prática médica, verificou-se que o alto custo $(19,7 \%)$ e a existência de evidências clínicas claras sobre a melhor prática a ser adotada (18\%) foram os critérios mais prevalentes. A dispersão expressiva no tempo de permanência hospitalar foi também apontada como critério para realização de tais estudos por 10,8\% dos estabelecimentos que os realizam.

\section{Discussão}

Apesar de nos últimos anos, após a Lei no . 9.656/98 e a criação da ANS, ter se verificado uma melhoria na quantidade e qualidade de dados sobre o mercado de saúde suplementar, ele ainda é pouco conhecido e considerado no planejamento do sistema de saúde no país. Essa questão assume grande relevância, em face da abrangência do setor, assim como da sua forte relação com o setor público 10.

De forma sintética e preliminar, este estudo evidenciou que a maior parte dos hospitais prestadores de serviços para operadoras era também provedora de serviços ao SUS, expressando claramente a necessidade de se alcançar maior integração, articulação e complementação entre os prestadores hospitalares públicos estatais e privados no atendimento ao SUS. Evidenciou ainda a expressiva sobreposição de operadoras na contratação de serviços de um mesmo prestador 10 .
A maioria dos hospitais localiza-se nas regiões Sudeste e Sul, acompanhando o padrão de distribuição de operadoras e de beneficiários de planos de saúde 11, bem como do próprio desenvolvimento regional; adicionalmente, ambas as regiões concentram os hospitais de maior porte e complexidade assistencial. Esse padrão seria esperado tendo em vista a lógica privada das operadoras de planos de saúde, mas se constitui em um desafio para o SUS, fortemente dependente da prestação de serviços hospitalares do setor privado, se considerada a perspectiva de assegurar a prestação de serviços hospitalares de forma mais equânime para o conjunto da população brasileira.

Mesmo que a UNIMED não se constitua em uma única empresa, merece destaque a importância das cooperativas médicas, em virtude de sua abrangência e sua capilaridade nacional; em adição, constituem-se nas principais provedoras de serviços hospitalares na saúde suplementar. Também são expressivas as participações da CASSI e GEAP, respectivamente, segunda e terceira operadoras em volume de faturamento e de internações para a maioria dos hospitais.

Deve ainda ser sublinhada a forte concentração do faturamento de cada hospital em algumas poucas operadoras, expressa, por exemplo, no fato da principal operadora, em volume de faturamento e de internações dos hospitais corresponderem, em média, a 52,3\% dos recursos provenientes dos planos de saúde. Isso sugere, também, uma concentração do mercado de planos de saúde, o que já foi apontado por outros estudos 10,12.

Observou-se baixa presença de estruturas e práticas de qualificação da gestão e de monitoramento da qualidade assistencial, endossando outros achados em estudos já realizados sobre hospitais brasileiros 8,9. Evidenciaram-se, porém, melhores resultados entre os hospitais que não prestam serviços ao SUS, sendo somente prestadores da saúde suplementar.

Especificamente no que concerne às práticas de gestão da clínica, pode surpreender em algum grau o fato de $51,6 \%$ dos hospitais referirem utilizar diretrizes clínicas, mas esse resultado parece ser validado na sua distribuição por complexidade assistencial. Vale ainda destacar tratar-se de um resultado geral, que não discrimina a abrangência de uso das diretrizes nos diversos serviços hospitalares e nem a fonte dessas diretrizes, não informando, portanto, sobre seu uso mais profissionalizado.

O conhecimento das características assistenciais de hospitais ou outros estabelecimentos de saúde provê subsídios para a constituição de redes de serviços capazes de dar respostas mais efi- 
cientes à demanda colocada pelos seus usuários. Adicionalmente, a possibilidade de se avançar na comparação da qualidade e dos preços praticados por tais serviços, especialmente os dos hospitais, constitui-se em um desafio que, potencialmente, propicia maior transparência e escolha mais qualificada, por gestores de sistemas ou microssistemas de saúde e por seus usuários, daqueles que melhor atendem as suas necessidades e expectativas 13,14,15.

A qualidade dos serviços de saúde constituise em forte preocupação presente nos diversos sistemas de saúde do mundo, sejam eles predominantemente públicos ou privados, sendo ainda insuficiente o conhecimento sobre a efetividade das estratégias destinadas à sua melhoria 16,17.
Em países mais desenvolvidos, onde a compra de serviços de saúde ocorre de forma mais criteriosa e a escolha do prestador público ou privado é orientada pelo seu desempenho - padrões pré-estabelecidos de estruturas, práticas e resultados assistenciais -, tem sido percebida uma melhoria da qualidade e uma diminuição dos custos da atenção ${ }^{15}$. A experiência internacional tem evidenciado ser fundamental incrementar a prestação de contas - accountability - e associar o repasse de recursos e outros incentivos a padrões pré-estabelecidos e desejados de desempenho dos prestadores, a fim de contribuir para a melhoria da qualidade dos serviços prestados e para uma utilização mais racional dos recursos existentes 15 .

\section{Resumo}

Este trabalho objetivou caracterizar os prestadores de serviços hospitalares no setor de saúde suplementar, uma vez que o conhecimento sobre tais prestadores ainda é incipiente, particularmente em relação à estrutura e à qualidade assistencial. Foi realizado um inquérito nacional, tendo como universo de interesse 3.817 hospitais. Uma amostra estratificada de 83 hospitais foi selecionada. A coleta de dados ocorreu entre setembro e dezembro de 2006, por meio de entrevistas com os dirigentes dos hospitais. Para a caracterização assistencial dos hospitais, foram contempladas variáveis relativas à capacidade instalada e produção de serviços, bem como às práticas e estruturas de garantia e monitoramento da qualidade do cuidado. A amostra final pesquisada resultou em 74 hospitais, correspondendo a um universo estimado de 3.799 unidades. Os hospitais prestadores de serviços para operadoras, majoritariamente provedores de serviços ao Sistema Unico de Saúde (SUS), apresentaram baixa presença de estruturas e práticas de qualificação da gestão e de monitoramento da qualidade assistencial.

Assistência Hospitalar; Qualidade da Assistência à Saúde; Saúde Suplementar

\section{Colaboradores}

M. C. Portela e S. M. L. Lima participaram do desenho do estudo, das análises e da redação do manuscrito. M. A. D. Ugá colaborou na coordenação da pesquisa, no desenho do estudo, nas análises e na revisão do manuscrito. M. T. L. Vasconcellos fez o desenho amostral, redigiu a parte relativa ao desenho amostral na seção de métodos e revisou o artigo. S. Gerschman contribuiu no desenho do estudo, nas análises e na revisão do manuscrito.

\section{Agradecimentos}

Os autores agradecem à Agência Nacional de Saúde Suplementar (ANS) e à Organização Pan-Americana da Saúde (OPAS/OMS) o apoio para a realização do estudo no qual este artigo se baseia.

Os autores agradecem ao Prof. Miguel Murat Vasconcellos, que fez parte da equipe do projeto e participou da concepção da pesquisa que originou este artigo, mas, infelizmente, faleceu antes de sua conclusão. 


\section{Referências}

1. Agência Nacional de Saúde Suplementar. Caderno de informação da saúde suplementar: beneficiários, operadoras e planos. Rio de Janeiro: Agência Nacional de Saúde Suplementar; 2008.

2. Hedlin D. A procedure for stratification by an extended Ekman rule. J Off Stat 2000; 16:15-29.

3. Haldane JBS. On a method of estimating frequencies. Biometrika 1945; 33:222-5.

4. Vasconcellos MTL, Silva PLN, Szwarcwald CL. Sampling design for the World Health Survey in Brazil. Cad Saúde Pública 2005; 21 Suppl 1:S89-99.

5. Hájek J. Limiting distribution in simple random sampling from a finite population. Publications of the Mathematics Institute of the Hungarian Academy of Science 1960; 5:361-74.

6. Field MJ, Lohr KN, editors. Clinical practice guidelines: directions for a new program. Washington DC: National Academy Press; 1990.

7. Mendes EV. Os sistemas de serviços de saúde: o que os gestores deveriam saber sobre essas organizações complexas. Fortaleza: Escola de Saúde Pública do Ceará; 2002.

8. Portela MC, Lima SML, Barbosa PR, Vasconcellos MM, Ugá MAD, Gerschman S. Caracterização assistencial de hospitais filantrópicos no Brasil. Rev Saúde Pública 2004; 38:811-8.

9. Lima SML, Barbosa PR, Portela MC, Ugá MAD, Vasconcellos MM, Gerschman S. Caracterização gerencial dos hospitais filantrópicos no Brasil. Cad Saúde Pública 2004; 20:1249-61.
10. Albuquerque C, Piovesan MF, Santos IS, Martins ACM, Fonseca AL, Sasson D, et al. A situação atual do mercado de saúde suplementar no Brasil e apontamentos para o futuro. Ciênc Saúde Coletiva 2008; 13:1421-30.

11. Portela MC, Lima SML, Ferreira VMB, Escosteguy CC, Brito C, Vasconcellos MTL. Diretrizes clínicas e outras práticas voltadas para a melhoria da qualidade assistencial em operadoras de planos de saúde sob a perspectiva dos seus dirigentes, no Brasil. Cad Saúde Pública 2008; 24:253-6.

12. Reis COO. Os desafios da ANS frente à concentração dos planos de saúde. Ciênc Saúde Coletiva 2007; 12:1041-50.

13. Jha AK, Li Z, Orav EJ, Epstein AM. Care in U.S. hospitals: the Hospital Quality Alliance Program. N Engl J Med 2005; 353:265-74.

14. Tynan A, Liebhaber A, Ginsburg PB. A health plan work in progress: hospital-physician price and quality transparency. Res Briefs 2008; (7):1-8.

15. Waters HR, Morlock LL, Hatt L. Quality-based purchasing in health care. Int J Health Plann Manage 2004; 19:365-81.

16. De Vos M, Graafmans W, Kooistra M, Meijboom B, Van Der Voort P, Westert G. Using quality indicators to improve hospital care: a review of the literature. Int J Qual Health Care 2009; 21:119-29.

17. Glickman SW, Ou FS, DeLong ER, Roe MT, Lytle BL, Mulgund J, et al. Pay for performance, quality of care, and outcomes in acute myocardial infarction. JAMA 2007; 297:2373-80.

Recebido em 06/Jun/2009

Versão final reapresentada em 27/Out/2009

Aprovado em 14/Dez/2009 